with anomalously intense radio emissions in the hope of learning more about the nature of these objects and the sources of their energy. There are also to be studies of the fine structure of many radio galaxies, with particular reference to their brightness distribution spectrum and magnetic field structure. According to Dr Lev Gindilis, who has been concerned with planning the RATAN sky surveying activities, these are to be conducted on a systematic basis and at the highest frequencies possible. This is prompted by the paucity of information in this range and the hope of observing quasars at the initial stage of their evolution. Gindilis considers RATAN to be an optimal instrument for surveys in the entire range from $4 \mathrm{~mm}$ to $20 \mathrm{~cm}$, in which there is a minimum of natural interference for observing cosmic sources. RATAN sky surveying work will also take in account the wishes of radio astronomers interested in the problem of communication with extraterrestrial intelligence (CETI).

Ultimately the RATAN control complex is to be linked to the main radioastronomical centres of the Soviet Union, with the result that the potential of this giant instrument will be used more effectively. RATAN-600 is also seen by Soviet radio telescope designers as a stepping stone towards far more powerful facilities.

\section{Aspects of plasmid behaviour}

from J. R. Saunders

A NATO Advanced Study Institute on the Biology of Bacterial Plasmids was held at Kavouri, $\mathrm{Nr}$ Athens from July 5-16, 1976.

INTEREST in the biology of bacterial plasmids has been stimulated both by their use as cloning vehicles for genetic engineering and by the increasing knowledge that a multitude of important bacterial properties are plasmid specified. The role of $R$ plasmids in mediating antibiotic resistance in clinically important bacteria is well known. Bacterial pathogenicity for plants can, however, also be encoded by plasmids. N. Van Larebeke (University of Ghent) showed that the ability of Agrobacterium tumifaciens to cause "crown gall" tumours in wounded plant tissues is determined by tumour-inducing (Ti) plasmids. Furthermore tumorigenic ability can be transmitted to other Agrobacteria either by transformation or by conjugation mediated by the $\mathbf{R}$ plasmid RP4. M. Holsters from the same group demonstrated that $\mathrm{Ti}$ plasmids allow host bacteria to synthesise and utilise either nopaline or octopine. It is interesting that tumours produced by $\boldsymbol{A}$. tumifaciens contain, (depending on the strain) either of these unusual amino acids, suggesting that certain plasmid genes may be expressed in tumour cells.

P. Williams (University of Wales, Bangor) hailed the 'degradative' plasmids of Pseudomonas, unlike most plasmids, as being totally beneficial to the biosphere as a whole. The ability of some Pseudomonas species to degrade and utilise many natural and synthetic organic substrates is due in part to the existence of plasmids determining the enzymes of specific catabolic pathways. The manipulation of these plasmids could therefore be used to construct bacterial strains capable of 'mopping-up' oil spillages and industrial wastes.

The translocation of resistance genes continues to generate interest. S. N. Cohen (Stanford University) stated that Transposon A ( $\operatorname{Tn} A)$ encoding resistance to ampicillin comprises part of the larger transposon TnS which specifies resistance to ampicillin, streptomycin and sulphonamides and that TnA may provide the driving force for the translocation of the larger unit. Evidence for interactions between transposons was also presented by $M$. H. Richmond (University of Bristol). $\mathrm{He}$ reported that the presence of $\operatorname{Tn} A$ on a plasmid reduces the frequency of transposition of a second copy of $\mathrm{TnA}$ to it by a factor of 10,000 in recombination-deficient $\left(\mathrm{rec}^{-}\right)$cells. On the other hand, in $\mathrm{rec}^{+}$cells the presence of a resident copy of TnA merely reduces the frequency of accretion of a second copy by $90 \%$. In the latter case however the incoming copy of $\mathrm{TnA}$ apparently replaces the resident $\mathrm{TnA}$ probably by reciprocal recombination rather than transposition. The results therefore suggest that one transposon in some way interferes with the recindependent translocation of like elements. The as yet unpublished work of several laboratories on the genetic structure and nature of proteins required for transposition may help to elucidate these phenomena.

J. Davies (University of Wisconsin) and R. Hermann (University of Heidelberg) advocated the use of $\lambda$ and fd phages respectively as host molecules for studying transposons. Phages possess the distinct advantage in that generally their genetical and physical properties, for example the location of promoters and partial known base sequences, are better characterised than those of plasmids. The ability to synthesise antibiotic-modifying enzymes in vitro, described by G. Hogenauer (Sandoz, Vienna) should also help our understanding of the structure and operation of resistance genes.

A major problem in genetic engineering is the expression of eukaryotic genes in prokaryotes (see News and Views, 262, 256; 1976). D. H. Gelfand (University of California, San Francisco) described the construction of plasmids carrying inserted Xenopus laevis 28S rDNA "downstream" of the operator-promoter region and part of the $\beta$-galactosidase gerie of the lactose operon. Induction of the lac operon in cells carrying such plasmids causes increased production of RNA complementary to rDNA. Furthermore, some derivatives with inserted genes produce $\beta$-galactosiclase with reduced enzymic activity but increased molecular weight. The production of polypeptides fused to this enzyme suggests that it may be possible to 'translate' some foreign genes in bacteria.

Potential vectors for natural spread of genes between bacterial genera are plasmids of the $\mathrm{P}$ incompatibility group (Datta et al., J. Bact., 108, 1244; 1971). P group plasmids are characterised by an extraordinary ability to transfer themselves to a wide range of Gram negative bacteria. They are thus attractive research tools for studying intergeneric gene transfer (see, for example, News and Views, 260, 191; 1976) and for the promotion of chromosomal gene transfer in species which lack their own sex factors. They could in addition contribute to the spread of resistance genes by crossing species barriers and depositing transposons in new hosts. V. Stanisich (University of Bristol) has also found that genes of plasmids normally restricted to Pseudomonas aeruginosa can be transferred to and expresed in $E$. coli by a technique essentially involving the formation of genetic hybrids with $\mathrm{P}$ group plasmids.

What then confers on these plasmids their unusual capacity for intergeneric transfer? One possibility is that their conjugation mechanism and specificity of attachment of $P$ group sex pili is unaffected by the nature of recipient cells. D. R. Helinski (University of California, San Diego) pointed out that $\mathbf{P}$ group plasmids not only exhibit a paucity of cut sites for many restriction endonucleases, but also that their replication machinery may be more complex and hence less dependent on host chromosome function than other plasmids. This could endow them both with some immunity against the battery of restriction enzymes likely to be encountered in intergeneric transfer and with the ability to replicate in a wide range of cellular environments. Accordingly the ability of $\mathbf{P}$ group plasmids to cross interspecies barriers in bacteria could well make them mediators of 'natural' genetic engineering. 